\title{
Organizational and team-based empathy: enhancing medical ethics consultation
}

Keywords: ironic, bioethics, ethics, healthcare, physicians

\section{Commentary}

It is somewhat ironic that research-supported end-of-life care is being politicized in the United States at a time when both major political parties jockey over which camp can craft the most affordable, accessible and high quality health care bill. Certainly, hospital based bioethics committees are not death panels. In fact, most do not have decision-making power. However, an awkward juxtaposition does exist between evidence-based healthcare practice and the overall financial burden to our healthcare system by providing care that is of little or no value. Most striking is that $20 \%$ of all Medicare spending occurs in the last two months of a patient's life. ${ }^{1}$ Superimpose this datum on the practice of an interdisciplinary ethics team and it creates an uncomfortable and challenging scenario.

\section{Organizational and team-based empathy}

While the literature offers many examples of team development, the concept of organizational and team empathy has yet to be developed. Defined here as an understanding of practice environment one occupies, organizational and team-based empathy is inclusive of one's ability to assess the organization or team and, subsequently, align professional skills with the needs of the organization. These activities, if successful, should allow for more productive team-based medical ethics consultation.

Most health care professions have a culture and a brand. Each interdisciplinary member of the ethic's team has been professionally prepared and socialized with a different set of values and ideology. Within the framework of the working team, each professional will now need to slightly "un-become" from their professional upbringing to best empathize with the practice orientations, stresses and accountabilities of their teammates.

For example, social workers are often the professionals caught among family system denial, physician reluctance to transition to hospice (or even palliative care) and administration pressure regarding patient flow and length of stay. Physicians assume the liability risk for most practice decisions and outcomes, while administration is accountable to the continuing management of resources. Each of these professions, plus many others, need to do a more purposeful job of organization and team (in this example) empathy.

\section{Conclusion and implications: team-based enhancement}

Gawande ${ }^{2}$ writes that while scientific breakthroughs have allowed people to live longer than at any other time in history, the aging and dying process have turned into a more medically dominated versus natural life experience. He honestly addresses critical issues such as physician lack of training and subsequent failings during a patient's dying process; yet, excludes the challenges this places on
Volume I Issue 3 - 2017

\author{
Ed Silverman \\ Keuka College, Chair Social Work, USA
}

Correspondence: Ed Silverman, Keuka College, Chair Social Work, USA, Tel 585-733-7065, Email esilverm@keuka.edu

Received: July 25, 2017| Published: August 15, 2017

other healthcare team members. Though Gawande's book focuses on physician practice, members of a typical ethics team can clearly see themselves.

Though obvious in retrospect, no one profession, regardless of status, can excel at all things. Superimpose the following sources of potential conflict on the end-of life care process, and this becomes quite clear: cultural diversity issues, lack of trust with the health care system, psychological issues and communication issues. These potential barriers to patient-care may be difficult to differentiate and identify by a given professional discipline. For example, Silverman ${ }^{3}$ writes that what can be perceived as a family's psychological denial to a grim prognosis is, many times, more a communication issue, or an unresolved crisis reaction or simply an unexpressed distrust of the health care system. A high functioning team that can empathize with not only the patient system, but with each other, can best maximize potential for a positive outcome.

Teamwork requires trust, productive conflict management, vulnerability and awareness of the task at hand. ${ }^{4}$ This commentary suggests that to maximize this process, one should understand and incorporate a degree of organizational and team-based empathy.

\section{Acknowledgements}

This is to acknowledge my permission to potential publish the above Commentary in you Journal.

\section{Conflict of interest}

The author declares no conflict of interest.

\section{References}

1. Friedman TL, Mandelbaum M. That use to be us. Farrar, New York, USA: Strauss and Giroux; 2011. 380 p.

2. Gawande A. Being mortal. New York, USA: Henry Holt and Company; 2014.

3. Silverman E. Barriers to effective end-of life transitional care: Challenges facing the emerging health care social worker. The New Social Worker, Fall, New York, USA: Springer; 2015. p. 26-28.

4. Lencioni P. The ideal team player. New Jersey, USA: The New Social Worker; 2016. 240 p. 